\title{
È possibile ridurre le complicanze infettive dei CVC per dialisi? Se sì, come?
}

\author{
Andrea Cavalli, Giuseppe Pontoriero \\ S.C. Nefrologia e Dialisi, Ospedale “Alessandro Manzoni”, Lecco
}

Is IT POSSIBLE TO REDUCE INFECTIOUS COMPLiCATIONS IN CVC FOR DiALYSIS? IF SO, HOW?

Abstract. The number of patients using a central venous catheter (CVC) for hemodialysis is too high, being far above $10 \%$ also in Italy. There are several complications associated with CVC use, among which infectious events are the most important, since they are related with relevant morbidity and mortality.

A recent meta-analysis, performed by Zhao, has shown that, considering the incidence of CVC-related infections, the citrate lock was better than heparin when used in association with antimicrobial agents (such as gentamicin or taurolidine) and at low to moderate concentration. Moreover, citrate use reduced bleeding episodes, while there were no differences in terms of exit-site infection and the use of thrombolytic treatment.

Results from a recent comparative effectiveness evaluation using a prospective, cluster-randomized design have underlined the importance of an adequate care of CVC and exit-site. The use of $2 \%$ chlorhexidine with $70 \%$ alcohol swab sticks for exit-site care and $70 \%$ alcohol pads to perform "scrub the hub" resulted in a significant $20 \%$ lower rate of central line-associated infections compared to the controls; this was accompanied by a significantly lower need of antibiotic therapy and lower hospitalization rates for both sepsis and vascular access-related infections and complications.

Either way, new trials and techniques are urgently needed to further improve the prognosis of patients performing hemodialysis using a CVC.

Key words: Central venous catheter, Hemodialysis, Citrate, Catheter-related infection, Exit-site infection

Conflict of interest: None.

Financial support: None.

Accettato: 16 Marzo 2014

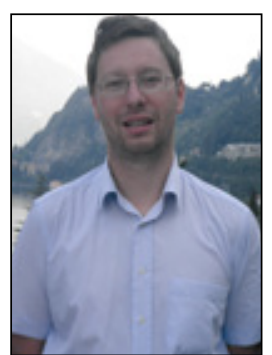

Andrea Cavalli
Secondo i dati del registro americano US Renal Data System (USRDS), l'incidenza di eventi infettivi negli emodializzati è aumentata del 43\% rispetto al 1993, rappresentando la seconda causa di ospedalizzazione e morte (dopo gli eventi cardiovascolari) (1). È ben intuibile come l'utilizzo del catetere venoso centrale (CVC) come accesso vascolare per emodialisi (HD) sia in gran parte responsabile dei suddetti dati, nonostante gli sforzi in atto per ridurne l'impiego (2). Negli USA, il 20\% dei pazienti effettua il trattamento emodialitico con CVC. Questa alta percentuale di CVC (oltre il doppio rispetto a quanto raccomandato dalla National Kidney Foundation-Kidney Disease Outcomes Quality Initiative) espone, con un'incidenza di 1.05 batteriemie ogni 1000 giorni di vita del CVC, ben 70.000 emodializzati al potenziale rischio di sepsi da infezione del CVC, che può determinare infezioni a distanza come osteomieliti, endocarditi, artriti settiche e ascessi e risultare fatale nel $12-25 \%$ dei casi (3).

Secondo i dati del censimento 2004, promosso dalla Società Italiana di Nefrologia, sul totale degli accessi vascolari, i CVC rappresentano il $12 \%$, contro il $5 \%$ delle protesi vascolari e 1'83\% delle fistole arterovenose native (4), ma gli ultimi dati del Dialysis Outcomes and Practice Patterns Study (DOPPS) 4 hanno mostrato un aumento di prevalenza dei CVC dal $10 \%$ del 2004 al 24\% del 2010 (5).

La valutazione di nuove strategie volte a ridurre le complicanze associate al CVC quale accesso vascolare per emodialisi rappresenta, pertanto, un "very hot topic" di terapia dialitica e, ultimamente, è stata oggetto di un intenso lavoro di ricerca che vogliamo riportare nella nostra rubrica $T N \& D$ Journal $C l u b$, collegandoci, peraltro, ad alcuni degli articoli pubblicati recentemente su questa Rivista $(6,7)$.

Prendendo in considerazione la modalità di chiusura del CVC, sembra evidente come la soluzione ideale debba presentare 
alcune caratteristiche: prevenire/ridurre gli episodi di sepsi correlati al CVC (almeno attraverso la via trans-luminale), essere efficace contro tutti gli organismi senza indurne resistenze e prevenire la formazione di trombi nel CVC. Partendo dall'evidenza che l'eparina possiede alcuni punti di debolezza, come il rischio di accidentale scoagulazione sistemica e la possibilità di promuovere in vitro la formazione del biofilm dello Staphylococcus aureus (8), sono state studiate altre soluzioni per la chiusura del CVC, come il citrato di sodio. L'azione anticoagulante di quest'ultimo è determinata dalla sua capacità di legarsi agli ioni calcio bloccando la cascata coagulatoria, oltre a presentare delle interessanti proprietà anti-microbiche (4). D'altra parte, vi sono alcune riserve sull'uso del citrato, legate alla possibilità di un suo effetto anti-coagulante sistemico (limitate, tuttavia, dalla sua rapida metabolizzazione in bicarbonato una volta passato in circolo, a eccezione dei soggetti con epatopatia) e all'eventuale rischio di aritmie, specialmente in caso di utilizzo ad alte concentrazioni (Tab. I).

Il gruppo cinese di Zhao et al. ha recentemente eseguito una revisione sistematica e una successiva meta-analisi dei trial randomizzati e controllati presenti in letteratura, che hanno confrontato il ruolo del lock del CVC con citrato di sodio rispetto all'utilizzo di eparina (9). Da un gruppo di 347 articoli, sono stati selezionati 13 studi randomizzati e controllati, svolti tra il 1998 e il 2012, in 1770 pazienti emodializzati, e un'esposizione al rischio di infezione da catetere della durata di 221.064 giorni (9 studi hanno considerato solo CVC tunnellizzati, 2 solo CVC non-tunnellizzati e 2 entrambi i CVC). Gli outcome primari valutati nella meta-analisi sono stati: l'incidenza di infezione sistemica correlata al CVC (intesa come una batteriemia senza evidenza di altra origine se non dal CVC), l'infezione dell'exitsite del CVC (cioè la presenza locale di secrezione purulenta $\mathrm{o}$ rossore), la rimozione del CVC per flusso ematico inadeguato e la necessità di un trattamento trombolitico.

L'incidenza di infezioni sistemiche correlate al CVC è risultata significativamente inferiore quando veniva utilizzato il citrato rispetto all'eparina (rischio relativo (RR) 0.39, intervallo di confidenza al 95\% (IC 95\%): 0.27-0.56, $\mathrm{P}<0.001$ ), anche se l'analisi di sottogruppi ha mostrato come il ricorso al citrato da solo dia risultati sovrapponibili a quelli ottenuti con l'eparina, mentre il vantaggio emergeva solo quando veniva utilizzato in associazione con gentamicina $(\mathrm{P}<0.001)$, taurolidina $(\mathrm{P}=0.003)$ o blu di metilene più metilparabene $\mathrm{e}$ propilparabene $(\mathrm{P}=0.008)$. Da un'ulteriore analisi, si evinceva che solo a una bassa (1.04-4\%) o moderata (4.6-7\%) concentrazione di citrato si ottenevano migliori risultati $(\mathrm{P}<0.001$ e $\mathrm{P}=0.003$ rispettivamente), mentre una sua elevata concentrazione (30-46.7\%) non mostrava benefici rispetto all'uso dell'eparina (9).

Per quanto riguarda l'incidenza di infezioni dell'exit-site, valutata in 8 (su 13) studi della meta-analisi, l'utilizzo del citrato ha mostrato solo una tendenza alla riduzione degli eventi infettivi rispetto all'eparina, ma non statisticamente significativa (RR 0.73, IC 95\%: 0.46-1.16, P = 0.2), così come non vi erano differenze di outcome anche nell'analisi di sottogruppi (citrato in associazione e a diversa concentrazione) (9).

Non sono state identificate differenze significative tra le due modalità di chiusura dei $\mathrm{CVC}$, anche per quanto riguarda la
TABELLA I - VANTAGGI E SVANTAGGI LEGATI ALL'UTILIZZO DEL CITRATO DI SODIO PER LA CHIUSURA DEL CVC PER EMODIALISI

\begin{tabular}{ll}
\hline \multicolumn{1}{c}{ Vantaggi } & \multicolumn{1}{c}{ Svantaggi } \\
\hline $\begin{array}{l}\text { Rapida metabolizzazione } \\
\text { a bicarbonato in caso di } \\
\text { passaggio sistemico }\end{array}$ & $\begin{array}{l}\text { Rischio di aritmie (da ipocalcemia), } \\
\text { in caso di utilizzo ad alte } \\
\text { concentrazioni }\end{array}$ \\
$\begin{array}{l}\text { Efficacia pari all'eparina nel } \\
\text { mantenere la pervietà dei CVC }\end{array}$ & $\begin{array}{l}\text { Cautela nell'utilizzo negli } \\
\text { epatopatici }\end{array}$ \\
Proprietà anti-microbiche & \\
\hline
\end{tabular}

TABELLA II - MESSAGGI DERIVANTI DALLA META-ANALISI DI ZHAO
1. Lock del CVC con solo citrato non superiore all'eparina in termini di prevenzione delle infezioni correlate al CVC
2. Lock del CVC con citrato in associazione con sostanze anti-microbiche superiore all'eparina in termini di prevenzione delle infezioni correlate al CVC
3. Nella prevenzione delle infezioni correlate al CVC, prediligere concen- trazioni basse-moderate di citrato rispetto ad alte concentrazioni
4. Minor numero di episodi di sanguinamento quando si utilizza il citrato rispetto all'eparina
5. Nessuna differenza tra citrato ed eparina nel prevenire le infezioni dell'exit-site del CVC
6. Nessuna differenza tra citrato ed eparina nel preservare la pervietà del $\mathrm{CVC}$

necessità di rimuovere il CVC per la presenza di un flusso inadeguato e la necessità di ricorrere a un trattamento trombolitico per il suo malfunzionamento $(\mathrm{P}=0.05$, cioè al limite della significatività statistica, in caso di utilizzo di citrato + gentamicina vs eparina) (9).

Due dei 13 studi analizzati nella meta-analisi hanno valutato anche l'incidenza di episodi di sanguinamento (outcome secondario), evidenziando una maggiore sicurezza del citrato rispetto all'eparina (RR 0.48, IC 95\%: 0.30-0.76, $\mathrm{P}=0.002$ ). Per tutti gli altri outcome secondari considerati (mortalità per tutte le cause, sopravvivenza dell'accesso vascolare senza episodi infettivi, trombosi e durata del CVC), non sono emerse differenze significative tra i due bracci di trattamento (9).

Pertanto, in termini di prevenzione delle infezioni correlate al CVC, il lock del CVC con citrato è risultato migliore rispetto all'eparina quando viene utilizzato in associazione con sostanze dotate di attività anti-microbica (gentamicina o taurolidina, per esempio) e a concentrazioni bassemoderate, come suggerito anche dall'American Society of Diagnostic and Interventional Nephrology e dalla European Renal Best Practice (10,11). L'utilizzo del citrato sembra, inoltre, ridurre gli episodi di sanguinamento, mentre non sono emerse differenze in termini di incidenza di infezioni dell'exit-site e di mantenimento della pervietà del CVC. La Tabella II riassume i messaggi derivanti dalla meta-ana- 
lisi di Zhao.

Occorre, tuttavia, sottolineare come tutti gli studi presi in considerazione dalla meta-analisi siano stati condotti in Paesi occidentali, limitandone, pertanto, in parte l'applicabilità dei risultati emersi (9).

Oltre alla valutazione della migliore modalità per effettuare il lock del CVC, altre procedure possono determinare un aumento (o una riduzione) degli episodi infettivi correlati ai CVC e, in particolare, quelle riguardanti la disinfezione dell'emergenza (exit-site) e lo "strofinamento" del CVC. A tal proposito, numerosi interventi sono stati riportati in letteratura nel corso degli ultimi anni (12-15), tra cui il progetto iniziato nel 2011 nei Centri Fresenius del Nord America e pubblicato recentemente (15). Quest'ultima esperienza rappresentava un progetto di miglioramento della qualità nell'ambito dell'aggiornamento delle procedure di gestione del CVC ispirate alle raccomandazioni congiunte di "US Center for Disease Control" (CDC) (14) e "Association for Professionals in Infection Control" (15). Nello specifico, si voleva valutare l'uso della clorexidina gluconata al $2 \%$ in associazione con bastoncini con alcol al $70 \%$ per la disinfezione dell'exit-site del CVC, in accordo con le raccomandazioni del CDC e con i risultati di una nota meta-analisi, che mostrava come la clorexidina riducesse del $49 \%$ il rischio di infezioni CVC-correlate rispetto allo iodopovidone (16). Il protocollo prevedeva l'utilizzo dei tamponi sterili con alcol al 70\% per eseguire lo "sfregamento del CVC" all'inizio della dialisi, alla fine e ogni volta che le linee del CVC venivano disconnesse durante il trattamento, prevedendo 15 secondi per l'esecuzione della procedura (17), ottenendo l'evidente vantaggio di minimizzare il contatto mani-CVC e di eliminare l'uso di liquido disinfettante per le mani richiesto per l'applicazione delle garze all'emergenza. L'obiettivo era, pertanto, quello di concentrarsi su strategie che permettessero la riduzione della colonizzazione del CVC sia per via intraluminale (corpo del CVC) sia per via extra-luminale (exit-site). Il progetto Fresenius è stato disegnato come un trial prospettico e randomizzato per gruppi di centri dialisi, suddivisi sulla base di zona geografica, dimensioni del centro e incidenza di emocolture positive a partenza dal CVC nel primo quadrimestre del 2011. In ogni coppia di centro dialisi, una struttura veniva randomizzata all'implementazione delle nuove procedure, mentre l'altra proseguiva nell'applicazione delle precedenti modalità (che, nella maggioranza dei casi, consisteva in iodo-povidone con o senza l'aggiunta di soluzioni a base di ipoclorito di sodio per la disinfezione dell' exit-site e del CVC, senza prevedere lo "strofinamento" del CVC) (15).

Lo studio prevedeva un basale, da Aprile a Giugno 2011, e una prima fase di follow-up, da Agosto a Ottobre 2011 (escludendo il mese di Luglio, in cui, storicamente, si raggiungono le più elevate incidenze di infezioni da $\mathrm{CVC}$ ), poi estesa ai successivi tre trimestri, per valutare se eventuali differenze emerse nel primo periodo si mantenessero ulteriormente, anche considerando che i centri dialisi venivano poi incoraggiati e materialmente supportati per proseguire con le nuove procedure anche una volta terminato ufficialmente il trial (15).

Gli infermieri venivano istruiti in loco da esperti, prevedendo una valutazione delle competenze acquisite al termine della formazione, oltre che controlli casuali e non annunciati da
TABELLA III - MESSAGGI DERIVANTI DALL'APPLICAZIONE DELLE PROCEDURE "SPERIMENTALI" DI GESTIONE DEL CVC NEL TRIAL DI ROSENBLUM

1. Minore incidenza di infezioni CVC-correlate (-22\% rispetto ai controlli)

2. Minore necessità di somministrazione di antibiotici per via endovenosa $(-20 \%$ rispetto ai controlli)

3. Minore ospedalizzazione correlata all'accesso vascolare

4. Minore ospedalizzazione secondaria ad episodi settici

5. Possibilità di ridurre sensibilmente il contatto mani-CVC e l'utilizzo di garze

6. Necessità di eseguire la disinfezione dell'emergenza prima di iniziare la seduta, comportando l'attesa di alcuni minuti-extra da parte del paziente

7. Rare reazioni avverse, con carattere locale: rossore, prurito, eritema o vescicole intorno all'emergenza, forse in parte secondari ad un inadeguato utilizzo della clorexidina

8. Utilità di eseguire una valutazione iniziale per testare un'eventuale allergia alla clorexidina

parte dei gruppi di controllo-qualità e di controllo-infezioni per monitorare le pratiche adottate e promuoverle.

L'endpoint primario dello studio era rappresentato dal confronto dell'incidenza delle emocolture positive correlate al CVC (analizzate da un laboratorio centralizzato) tra i due gruppi di trattamento. Invece, gli endpoint secondari comprendevano: l'utilizzo di terapia antibiotica per via endovenosa, l'ospedalizzazione da infezione correlata al CVC o per sepsi e l'incidenza di reazioni avverse (15).

Sono stati randomizzati 422 centri dialisi (suddivisi in 211 paia), comprendenti 9160 pazienti portatori di CVC e più di 700.000 giorni di esposizione al CVC. Al basale, non vi erano differenze tra gruppo di trattamento e controllo in termini di incidenza di infezioni da CVC (rispettivamente 0.85 vs 0.86 per 1000 giorni di CVC), mentre, nel corso del follow-up, emergeva una differenza significativa: 0.81 nel gruppo sperimentale rispetto a 1.04 nei controlli, con un'evidente riduzione del $22 \%(\mathrm{P}=0.02)$. Vista l'assenza di cambiamenti rispetto al basale nel braccio sperimentale, le nuove procedure di disinfezione del CVC potrebbero, quindi, aver attenuato il trend di aumento delle infezioni CVC-correlate tipico dell'estate ed emerso, invece, nel braccio di controllo (15).

L'incidenza della somministrazione di antibiotici per via endovenosa, sovrapponibile al basale, nel gruppo sperimentale diventava inferiore del $20 \%$ rispetto ai controlli (2.53 vs $3.15 / 1000$ giorni di $\mathrm{CVC}, \mathrm{P}<0.001$ ), così come le ospedalizzazioni correlate all'accesso vascolare e secondarie alla sepsi erano inferiori del $38 \%$ e del $36 \%$ rispettivamente (15).

Sono state documentate 184 reazioni avverse in 82 pazienti $(2 \%)$, tutte con carattere locale: rossore, prurito, eritema o vesciche intorno all'emergenza. Tali eventi, correlati a reazioni cutanee da clorexidina, potrebbero essere in parte ritenuti secondari a un inadeguato utilizzo del disinfettante e, quindi, evitabili con maggiori attenzioni (per esempio, rispettandone i tempi di 
asciugatura). Come conseguenza, si raccomandava l'esecuzione di prove che valutassero l'eventuale allergia alla clorexidina, per eventualmente passare a un disinfettante alternativo (15).

Da parte dello staff infermieristico venivano riportati numerosi commenti positivi, come la semplicità dell'applicazione dei nuovi protocolli e la possibilità di evitare l'utilizzo di garze e di dover inzuppare i tappi del CVC e di ridurre il contatto mani-CVC. L'osservazione più frequente riguardava la necessità di eseguire la disinfezione dell'emergenza prima di iniziare la seduta, determinando l'attesa di alcuni minuti-extra da parte del paziente (15).

La Tabella III riassume i messaggi derivanti dall'applicazione delle procedure "sperimentali" di gestione del CVC nel trial di Rosenblum.

In un'analisi post-hoc del successivo periodo di follow-up, fino al Luglio 2012, si è evidenziato il mantenimento dei risultati ottenuti nella prima fase del trial, ottenendo una riduzione del $41 \%$ dell'incidenza di emocolture positive (da 0.86 a circa 0.50 per 1000 giorni di CVC) e del $27 \%$ del tasso di ospedalizzazione per sepsi. Questi dati si tramutano anche in un'evidente riduzione dei costi della spesa sanitaria, che permetterebbe, quindi, di giustificare e sostenere i maggiori costi derivati dall'utilizzo di clorexidina e tamponi con alcol, rispetto alle precedenti modalità.

Gli importanti risultati emersi dal trial di Rosenblum (15) sono, peraltro, stati confermati anche da altri dati e si inseriscono nel gruppo di attività che prevedono modifiche di pratiche cliniche a più livelli e che, se adeguatamente implementate, permettono di ottenere ripercussioni positive non solo in campo clinico, ma anche nel senso di appartenenza a uno staff motivato a un miglioramento continuo (15).

Abbiamo, pertanto, potuto capire come, nell'importante campo della prevenzione delle complicanze infettive correlate all'utilizzo del CVC per emodialisi, siano in atto numerosi sforzi che effettivamente possono determinare un miglioramento della prognosi dei nostri pazienti.

Ora occorre, quindi, che ognuno di noi rivaluti le strategie adottate dal proprio centro dialisi e imposti un programma di monitoraggio ed eventualmente delle modifiche, volte a perfezionare questo vitale (nel vero senso della parola!) aspetto dialitico.

\section{Riassunto}

Nonostante gli sforzi in atto, la percentuale di pazienti che utilizzano un catetere venoso centrale (CVC) per eseguire il trattamento emodialitico è attualmente troppo elevata e ben superiore al $10 \%$ anche in Italia. Le complicanze associate all'uso dei CVC sono numerose, ma le più temibili sono quelle infettive, responsabili di significative morbilità e mortalità. Una recente meta-analisi di Zhao ha riportato come, in termini di prevenzione delle infezioni correlate al CVC, il lock con citrato sia risultato migliore rispetto all'eparina, quando utilizzato in associazione a sostanze dotate di attività anti-microbica (gentamicina o taurolidina, per esempio) e a concentrazioni basse-moderate. Inoltre, l'utilizzo del citrato ridurrebbe gli episodi di sanguinamento, mentre non sono emerse differenze in termini di incidenza di infezioni dell'exit-site e di mantenimento della pervietà del CVC. I risultati emersi da un recente trial prospettico hanno sottolineato l'importanza di un'adeguata gestione dell'emergenza del CVC e del CVC stesso. Infatti, l'utilizzo di clorexidina al $2 \%$ per la disinfezione dell'exit-site e l'utilizzo di tamponi sterili con alcol al $70 \%$ per eseguire lo "sfregamento del CVC" permettevano di ridurre del $20 \%$ l'incidenza delle infezioni correlate al CVC, oltre a garantire una minore necessità di terapia antibiotica e una ridotta ospedalizzazione per sepsi e complicanze infettive CVC-correlate.

Tuttavia, sono necessari nuovi studi e provvedimenti per poter migliorare ulteriormente la prognosi dei pazienti che utilizzano un CVC come accesso vascolare per emodialisi.

Parole chiave: Catetere venoso centrale, Emodialisi, Citrato, Infezione correlata al catetere, Infezione dell'emergenza

Dichiarazione di conflitto di interessi: Gli Autori dichiarano di non avere conflitto di interesse.

Contributi economici agli Autori: Gli Autori dichiarano di non aver ricevuto sponsorizzazioni economiche per la preparazione dell'articolo.

Indirizzo degli Autori:

Prof. Giuseppe Pontoriero

S.C. Nefrologia e Dialisi

Ospedale "Alessandro Manzoni"

Via dell'Eremo 9/11

23900 Lecco

g.pontoriero@ospedale.lecco.it

\section{Bibliografia}

1. Collins AJ, Foley RN, Herzog C, et al. US Renal Data System 2012 Annual Data Report. Am J Kidney Dis 2013; 61 (1 Suppl. 1): e1-480.

2. Centers for Medicare \& Medicaid Services. The Fistula First Breakthrough Initiative. http://fistulafirst.org/. Accessed
December 20, 2012.

3. Centers for Disease Control and Prevention (CDC). Vital signs: central line-associated blood stream infections, United States 2001, 2008, and 2009. MMWR Morbid Mortal Wkly Rep 2011; 60 (8): 243-8.

4. Alloatti S, Strippoli GF, Buccianti G, Daidone G, Schena FP. Current structure and organization for renal patient assistance in 
Italy. Nephrol Dial Transplant 2008; 23: 1323-9.

5. 2010 DOPPS Annual Report www.dopps.org/annualreport/index.htm.

6. Cornacchiari M, Gallieni M, Stasi A, Ponticelli MG, Gidaro B, Guastoni C. Prevenzione delle infezioni catetere venoso centrale correlate (CRBSI). Giornale di Tecniche Nefrologiche \& Dialitiche 2013; 25 (3): 220-4.

7. Montani A, Pozzi L, Granatieri L, Magugliani M, Fusè O, Moretti M. Protocollo per la prevenzione delle CRBSI. Giornale di Tecniche Nefrologiche \& Dialitiche 2013; 25 (3): 225-7.

8. Shanks RM, Sargent JL, Martinez RM, Graber ML, O'Toole GA. Catheter lock solutions influence staphylococcal biofilm formation on abiotic surfaces. Nephrol Dial Transplant 2006; 21 (8): 2247-55.

9. Zhao Y, Li Z, Zhang L, et al. Citrate versus heparin lock for haemodialysis catheters: a systematic review and meta-analysis of randomized controlled trials. Am J Kidney Dis 2013; 63 (3): 479-90.

10. Moran JE, Ash SR. Locking solutions for hemodialysis catheters; heparin and citrate-a position paper by ASDIN. Semin Dial 2008; 21 (5): 490-2.

11. Vanholder R, Canaud B, Fluck R, et al. Catheter-related blood stream infections (CRBSI): a European view. Nephrol Dial Transplant 2010; 25 (6): 1753-6.

12. Battistella M, Bhola C, Lok CE. Long-term Follow-up of the Hemodialysis Infection Prevention With Polysporin Ointment (HIPPO) Study: A Quality Improvement Report. Am J Kidney Dis 2011; 57 (3): 432-41.
13. Lindberg C, Downham G, Buscell P, Jones E, Peterson P, Krebs V. Embracing collaboration: A novel strategy for reducing bloodstream infections in outpatient hemodialysis centers. Am J Infect Control 2013; 41: 513-9.

14. Patel PR, Yi SH, Booth S, et al. Bloodstream Infection Rates in Outpatient Hemodialysis Facilities Participating in a Collaborative Prevention Effort: A Quality Improvement Report. Am J Kidney Dis 2013; 62(2): 322-30.

15. Rosenblum A, Wang W, Ball LK, Latham C, Maddux FW, Lacson E Jr. Hemodialysis Catheter Care Strategies: A ClusterRandomized Quality Improvement Initiative. Am J Kidney Dis 2014; 63 (2): 259-67.

16. Rebmann T, Barnes SA. Preventing infections in hemodialysis: an executive summary of the APIC Elimination Guide. Association for Professionals in Infection Control and Epidemiology. Am J Infect Control 2011; 39 (1): 72-5.

17. O'Grady NP, Alexander M, Burns LA, et al. Healthcare Infection Control Practices Advisory Committee (HICPAC). Guidelines for the prevention of intravascular catheter-related infections. Clin Infect Dis 2011; 52 (9): e162-93.

18. Chaiyakunapruk N, Veenstra DL, Lipsky BA, Saint S. Chlorhexidine compared with povidone-iodine solution for vascular catheter-site care: a meta-analysis. Ann Intern Med 2002; 136 (11): 792-801.

19. Kaler W, Chinn R. Successful disinfection of needleless access ports: a matter of time and friction. J Assoc Vasc Access 2007; 12 (3): $140-7$. 